\title{
Seed germination and physiological responses of quinoa to selenium priming under drought stress
}

\author{
Shocofeh Gholami¹ (D), Majid Amini Dehaghi1,* (D), Alireza Rezazadeh² (D), Amir Mohammad Naji1 \\ 1. Shahed University rọ - Faculty of Agricultural Sciences - Department of Agriculture and Plant Breeding - Tehran, Iran. \\ 2. Shahed University rợ - Faculty of Agriculture - Department of Plant Protection - Tehran, Iran. \\ Received: Jun. 18, 2021 | Accepted: Nov. 3, 2021 \\ Section Editor: Hector Valenzuela \\ *Corresponding author: amini@shahed.ac.ir \\ How to cite: Gholami, S., Dehaghi, M. A., Rezazadeh, A. and Naji, A. M. (2022). Seed germination and physiological responses of quinoa to \\ selenium priming under drought stress. Bragantia, 81, e0722. https://doi.org/10.1590/1678-4499.20210183
}

\begin{abstract}
The early stages of quinoa germination are sensitive to drought stress. For this purpose, a study entitled the effect of selenium in different concentrations on germination characteristics and some antioxidant enzymes of quinoa under drought stress conditions with polyethylene glycol (PEG 6000) was investigated. The first experimental factor was seed priming with selenium (from two sources: sodium selenate and selenium nanoparticles: SeNPs $\approx 33.4 \mathrm{~nm}$ ) at $0.5,1.5,3,4.5,6 \mathrm{mg} \cdot \mathrm{L}^{-1}$ concentrations, besides, no priming treatment was used as control. The second factor was drought stress with PEG 6000 in concentrations $0,-0.4,-0.8$, and $-1.2 \mathrm{MPa}$. Drought stress with accumulation of reactive oxygen species (ROS) had a negative effect on most of the measured traits. In seeds that were primed with appropriate selenium concentrations, germination parameters and antioxidant enzyme activity as well as proline and protein content increased compared to the control treatment. Under conditions of severe stress ( $-1.2 \mathrm{MPa}$ ), the highest activity of catalase (CAT), superoxide dismutase (SOD), and ascorbate peroxidase (APX) enzymes was observed in prime with selenium nanoparticles at concentrations of 4.5 , 6.0 and $4.5 \mathrm{mg} \cdot \mathrm{L}^{-1}$, respectively. Concentrations higher than $3 \mathrm{mg} \cdot \mathrm{L}^{-1}$ of selenium nanoparticles and concentrations of $3 \mathrm{mg} \cdot \mathrm{L}-1 \mathrm{sodium}$ selenate had the highest accumulation of photosynthetic pigments under control (stress-free) conditions. The present study shows that selenium priming can reduce the harmful effects of drought stress on quinoa by altering germination properties and biochemical properties.

Key words: antioxidants, Chenopodium quinoa Willd., photosynthetic efficiency, ROS.
\end{abstract}

\section{INTRODUCTION}

Drastic changes in the climate, erratic precipitation, and rapid increases in global population have reduced crop production and endangered global food security. These changes will challenge the population's needs and demand in the future (Magwanga et al. 2018). Plants experience several environmental stresses. Drought stress is one of the most common stresses that can affect physiological processes such as photosynthesis, nutrient uptake and transport, and even grain yield due to plant species susceptibility and growth stage (Cai et al. 2020).

The excessive increase of reactive oxygen species (ROS) under drought stress conditions causes oxidative damage and eventually plant death (Qureshi et al. 2018). Therefore, plants show many responses to drought stress that depends on the type and plant species (Ul-Allah et al. 2018). Seed priming consists of controlled hydration and drying of seeds to enhance rapid germination and sustained establishment under stress conditions (Dawood 2018).

Priming the seeds with macro- and micronutrients during a period before sowing to improve the stand establishment and productivity of field crops is called nutripriming (Farooq et al. 2018; Shivay et al. 2016). The use of selenium to reduce ROS and increase antioxidant defense enhances plant growth and resistance to abiotic stresses such as drought stress (Hassan et al. 2020; Reis et al. 2017).

An advanced method to reduce the use of chemical fertilizers, changes in food production, increase nutrient efficiency, stress tolerance, and many challenges in agriculture is the use of nanotechnology (Manjunatha et al. 2016; Upadhyaya 
et al. 2017). The amount of selenium in food depends on the different forms of selenium in the soil and its absorption and accumulation in plants and herbivores (Hossain et al. 2021). Selenium nanoparticles have higher mobility and solubility, and more bioactivity and biosafety than soluble mineral salts Se, mainly selenite (Se (IV)) and selenate (Se (VI)). (Djanaguiraman et al. 2018; Xia et al. 2017).

Seed priming with selenium increases germination and radical elongation (Nawaz et al. 2013; Ullah et al. 2019). Selenium had a positive effect on rice seed germination and seedling growth (Moulick et al. 2017). Selenium also promotes plant growth and development under various stresses by increasing plants' resistance and antioxidant capacity (Chen et al. 2020). The application of selenium at low concentrations increased enzymatic and nonenzymatic antioxidants' activity, reducing the ROS in wheat (Sattar et al. 2019) and corn (Jiang et al. 2017). Selenium reduces lipid peroxidation by reducing ROS under drought stress conditions, thereby increasing leaf area, increasing transpiration efficiency, and improving photosynthesis under these conditions (Khan et al. 2015; Zhang et al. 2014). Application of selenium by increasing the activity of enzymatic antioxidants such as superoxide dismutase (SOD), catalase (CAT), catechol peroxidase (CPX), and nonenzymatic antioxidants such as ascorbate and carotenoids eliminates ROS and increases plant tolerance to various abiotic stresses (Reis et al. 2017; Silva et al. 2018). Catalase activity and proline content in wheat cultivars treated with selenium increased compared to the nonapplication of selenium (Sajedi and Madani 2018). Selenium application also increased proline accumulation in wheat seedlings under drought stress conditions (Chen et al. 2020). The application of chitosan-selenium nanoparticles enhanced the level of antioxidant in bitter melon under salt stress (Sheikhalipour et al. 2021). Also seed priming with silver nanoparticles considerably increased the activities of superoxide dismutase (SOD), catalase (CAT), peroxidase (POD) in pearl millet (Khan et al. 2020).

Selection of plants that require less inputs and are stress-tolerant with high nutritional value is a solution to improve food security in the face of the increasing global population (Delatorre-Herrera et al. 2019). Quinoa (Chenopodium quinoa Willd.) is a pseudocereal with high adaptability to adverse climatic and soil factors period due to its high levels of gluten-free protein, high fiber, complex carbohydrates, vitamins, carotenoids and high nutritional value quinoa has been introduced as an alternative product to improve food security (Graf et al. 2016; Gordillo-Bastidas et al. 2016; Pereira et al. 2019). This plant protects the body against many diseases, especially cancer and cardiovascular disease (Nowak et al. 2016). This study was performed with the hypothesis that selenium can reduce ROS under drought stress by increasing antioxidant activity and thus improving seedling emergence and growth.

\section{MATERIAL AND METHODS}

\section{Experimental design and seed material}

This research was conducted at the central laboratory of Shahed University of Tehran, Iran, in 2020. Fresh matured quinoa seeds of Giza 1 variety were obtained from the Seed Breeding and Seedling Institute's production fields of Karaj, Iran. The selenium nanoparticles used in this study were obtained from the NANOSANY Corporation (Mashhad, Iran). Sodium selenate (NaSeO4) and PEG 6000 were purchased from Merck (Darmstadt, Germany) Chemical Company. To assess the effect of seed priming with selenium (sodium selenate $\left(\mathrm{NaSeO}_{4}\right)$ ) and selenium nanoparticles (SeNPs $\approx 33.4 \mathrm{~nm}$ ) on germination indices and biochemical characteristics of seedlings under drought stress a factorial experiment was conducted based on a completely randomized design with three replications.

To assess water stress tolerance during germination, quinoa seeds were surface sterilized by $2.5 \%$ sodium hypochlorite solution for $3 \mathrm{~min}$ and then washed thoroughly with distilled water. Afterward, seeds were equally divided into six batches. Soaking of seeds was carried out by putting a constant number of seeds of each batch for $6 \mathrm{~h}$ in glass containers, each containing a continuous amount $(100 \mathrm{~mL})$ of $\mathrm{NaSeO} 4$ and selenium nanoparticle treatment solutions at $0.5,1.5,3,4.5$, $6 \mathrm{mg} \cdot \mathrm{L}^{-1}$ concentrations. Besides, a nonpriming treatment was used as control. At the end of the priming treatments, the seeds were washed with distilled water and air-dried for $24 \mathrm{~h}$. Then 100 seeds were placed in Petri dishes $(12 \mathrm{~cm})$ containing double filter paper (Whatman International Ltd., Maidstone, UK) and the drought stress treatments using $5 \mathrm{~mL}$ PEG 6000 at 
concentrations of 0 (distilled water [control]), $-0.4,-0.8$, and $-1.2 \mathrm{MPa}$ were added to the Petri dishes, for one week placed on a growth chamber with a controlled temperature of $25^{\circ} \mathrm{C}$., a light period of $16 \mathrm{~h}$ and $8 \mathrm{~h}$ of darkness (Hajihashemi et al. 2020) and relative humidity of 70\% (Panuccio et al. 2014).

At this stage, germination component was calculated according to ISTA (2008). Seed germination was recorded everyday according to AOSA (1990) until a constant number was reached. Seeds were considered germinated when the length of radicle and hypocotyl was more than $1 \mathrm{~mm}$. The following formula was used to compute the germination rate (Li et al. 2007; Liopa-Tsakalidi 2012; Thabet et al. 2018):

$$
\text { Germination rate }=(\text { number of germinated seeds } / \text { number of total seeds }) \times 100
$$

where GR = germinated seeds on day 7 / total seeds $100 \%$.

\section{Biochemical analysis}

The samples were randomly collected from each Petri dish 5 days after germination for determination of proline, total soluble proteins (TSP), photosynthetic pigments (chlorophyll a, b, and total, and carotenoids), and antioxidant enzymes (SOD, CAT, APX) using standard procedures.

\section{Photosynthetic pigments analysis}

Arnon's (1949) method were used to extract photosynthetic pigments, chlorophyll a, b, total chlorophyll, and carotenoids. For this purpose, about $1.0 \mathrm{~g}$ of seedling samples were immersed in liquid nitrogen for a few seconds to stop the metabolic processes of the tissue and then ground in $80 \%$ acetone. The ground mixture was centrifuged at $3000 \times \mathrm{g}$ for $10 \mathrm{~min}$. After centrifugation, the supernatant was collected and absorbance of the supernatant was measured using a UV-Vis spectrophotometer (Lambda 25, PerkinElmer, Inc., USA) at wavelengths of 663.2, 645, 470 and $510 \mathrm{~nm}$. The pigment content was estimated using the provided extinction coefficients and formulae and reported in $\mathrm{mg} \cdot \mathrm{g}^{-1} \mathrm{FW}$ :

$$
\begin{aligned}
& \text { Chlorophyll a }\left(\mathrm{mg} \cdot \mathrm{g}^{-1} \mathrm{FW}\right)=12.7\left(\mathrm{~A}_{663}\right)-2.69\left(\mathrm{~A}_{645}\right) \times \mathrm{V} / 1000 \times \mathrm{W} \\
& \text { Chlorophyll b }\left(\mathrm{mg} \cdot \mathrm{g}^{-1} \mathrm{FW}\right)=22.9\left(\mathrm{~A}_{645}\right)-2.69\left(\mathrm{~A}_{663}\right) \times \mathrm{V} / 1000 \times \mathrm{W} \\
& \text { Total chlorophyll }\left(\mathrm{mg} \cdot \mathrm{g}^{-1} \mathrm{FW}\right)=20.2\left(\mathrm{~A}_{645}\right)+8.02\left(\mathrm{~A}_{663}\right) \times \mathrm{V} / 1000 \times \mathrm{W} \\
& \text { Carotenoid }\left(\mathrm{mg} \cdot \mathrm{g}^{-1} \mathrm{FW}\right)=7.6\left(\mathrm{~A}_{470}\right)-14.9\left(\mathrm{~A}_{510}\right) \times \mathrm{VD} / 1000 \mathrm{~W}
\end{aligned}
$$

\section{Free proline assay}

The Bates et al. (1973) method was used to determine the amount of seedling tissue proline. First, $0.2 \mathrm{~g}$ of a fresh seedling sample and $10 \mathrm{~mL}$ of $3 \%$ sulfosalicylic acid were thoroughly ground in a porcelain mortar. The resulting homogeneous material was centrifuged at $13,000 \mathrm{rpm}$ at $4{ }^{\circ} \mathrm{C}$ for $10 \mathrm{~min}$. Then, $2 \mathrm{~mL}$ of the filtered extracts were transferred to capped tubes, and $2 \mathrm{~mL}$ of ninhydrin reagent and $2 \mathrm{~mL}$ of glacial acetic acid were added to all tubes. After closing the tubes, they were immersed in $100^{\circ} \mathrm{C}$ water for $1 \mathrm{~h}$. After cooling in an ice bath, $4 \mathrm{~mL}$ of toluene was added to each tube. The tubes were then vortexed for 15-20 s to be mixed. After 1-2 min, the red zinc phase, which contained toluene-soluble proline was removed, and, to mix the two solutions the tubes were shaken using a vortex for 15-20 s. Finally, the red phase, which turned red and contained toluene-soluble proline, was removed, and its absorbance was noted at $520 \mathrm{~nm}$ using a spectrophotometer (Hitachi, 220, Japan). The proline concentration was estimated on a basis using a standard curve as follows: 
$\mu \mathrm{mol}$ proline $\cdot \mathrm{g}^{-1} \mathrm{FW}=\left[\left(\mathrm{mg}\right.\right.$ of proline $\left.\cdot \mathrm{mL}^{-1}\right) \times(\mathrm{mL}$ toluene $) /($ wt. sample $\left./ 5)\right] / 115$

The Du and Bramlage (1994) method was used to measure the amount of SOD. The decrease in absorption of the superoxide-nitroblue tetrazolium complex caused by the enzyme was used to calculate its activity. The reaction mixture, which included $50 \mathrm{mmol} \cdot \mathrm{L}^{-1}$ potassium phosphate buffer $(\mathrm{pH} 7.8), 13 \mu \mathrm{mol} \cdot \mathrm{L}^{-1}$ of $0.1 \mathrm{~mol} \cdot \mathrm{L}^{-1}$ methionine, $75 \mu \mathrm{mol} \cdot \mathrm{L}^{-1}$ nitroblue tetrazolium chloride (NBT), $2 \mu \mathrm{mol} \cdot \mathrm{L}^{-1}$ riboflavin, $10 \mu \mathrm{mol} \cdot \mathrm{L}^{-1} \mathrm{EDTA}$, and $20 \mu \mathrm{mol} \cdot \mathrm{L}^{-1}$ of leaf enzyme extract, was incubated for $30 \mathrm{~min}$ under light and dark condition. A tube without enzyme extract was used as a control. Inhibition of photooxidation of NBT under fluorescent light in the presence of riboflavin and methionine was measured at $560 \mathrm{~nm}$ and expressed in units per mg of protein. One unit of enzyme activity was taken as the amount of enzyme that reduced the absorbance value of the samples by $50 \%$ compared to tubes without enzyme.

\section{Catalase (CAT) assay}

The Zhang and Kirkham (1994) method was used to measure CAT enzyme activity. First, $0.5 \mathrm{~g}$ of fresh seedling tissue was ground with $3 \mathrm{~mL}$ of phosphate buffer $(\mathrm{pH}=7)$ in a porcelain mortar and $0.1 \mathrm{~g}$ of the enzyme extract was mixed with $5.9 \mathrm{~mL}$ of $\mathrm{H} 2 \mathrm{O} 2$ after centrifugation to reduce the rate of adsorption. After $20 \mathrm{~s}$ at a wavelength of $240 \mathrm{~nm}$ CAT activity was calculated, expressed as unit $\mathrm{min} \cdot \mathrm{g}^{-1} \mathrm{FW}$.

\section{Ascorbate peroxidase (APX) assay}

Ascorbate peroxidase activity was measured using the Nakano and Asada (1981) method, on reduced adsorption at $290 \mathrm{~nm}$ (extinction coefficient equal to $2.8 \mathrm{~mm} \cdot \mathrm{cm}^{-1}$ ) in the reaction. Enzyme activity was determined by adding a solution containing $50 \mathrm{mmol} \cdot \mathrm{L}^{-1}$ of phosphate buffer $(\mathrm{pH} 7.0), 30 \mathrm{mmol} \cdot \mathrm{L}^{-1}$ of hydrogen peroxide, $10 \mathrm{mmol} \cdot \mathrm{L}^{-1}$ of ascorbate and $100 \mu \mathrm{L}$ of the enzymatic extract.

\section{Soluble protein assay}

Soluble protein content was determined by the Bradford (1976) method using bovine serum albumin as the standard. As a result, this assay involves the binding of Coomassie Brilliant Blue G-250 to aromatic amino acid radicals and the measurement of color at $595 \mathrm{~nm}$.

\section{Statistical analysis}

Distribution normality of data was determined according to the Kolmogorov-Smirnov and Shapiro-Wilk test. The studied traits (i.e., germination indices and physiological characteristics of seedlings) were analyzed using Statistical Analysis System software (SAS Institute, Cary, NC, USA, version 9.2). Differences between means were separated using Duncan's test at the 0.05 statistical probability level.

\section{RESULTS}

\section{Germination percentage}

This study showed that application of sodium selenate and selenium nanoparticles significantly $(\mathrm{p} \leq 0.01)$ improved the germination percentage of quinoa seeds under drought stress caused by PEG (Table 1). Data indicate that nutripriming with sodium selenate and selenium nanoparticles at concentrations of 0.5 to $4.5 \mathrm{mg} \cdot \mathrm{L}^{-1}$ at all stress levels significantly increased the germination percentage. However, priming with sodium selenate had a more positive effect on germination 
percentage than selenium nanoparticles. Thus, the highest germination percentage (96\%) was achieved with $4.5 \mathrm{mg} \cdot \mathrm{L}^{-1} \mathrm{of}$ sodium selenate under nonstress and the lowest germination percentage $(60 \%)$ was recorded with nonpriming under 1.2 MPa stress (Fig. 1).

$$
\square \mathrm{MP} \square-0.4 \mathrm{MPa} \quad \square-0.8 \mathrm{MPa} \quad \square-1.2 \mathrm{MPa}
$$

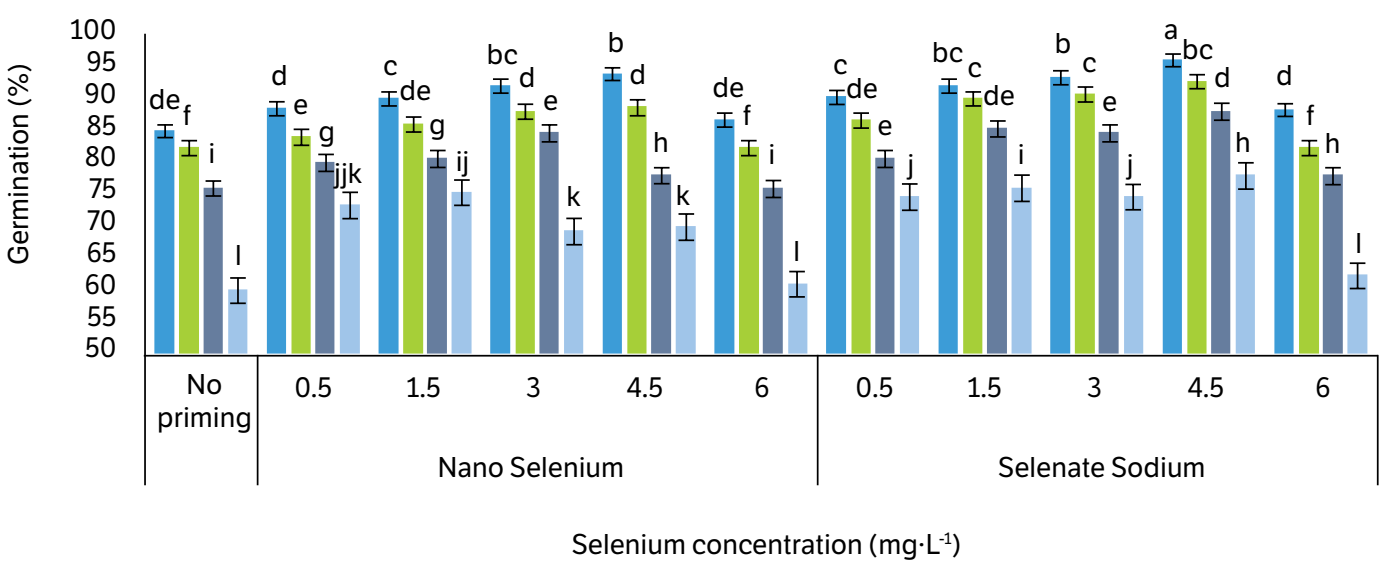

Figure 1. Comparison of different sodium selenate and selenium nanoparticles concentrations effects on germination percentage of quinoa under drought stress conditions.

Note. Bars labelled with the same letters are not significantly different according to Duncan’s multiple comparison test at $p<0.05$.

\section{Germination rates}

The data analysis showed that priming with selenium under drought stress and the interaction effects of these treatments was significant $(\mathrm{p} \leq 0.01)$ with respect to germination rate (Table 1 (. The germination rate decreased by increasing PEG concentration. With respect to selenium, concentrations higher than $4.5 \mathrm{mg} \cdot \mathrm{L}^{-1}$ reduced the germination rate. The highest germination rate (1.74 seed per day) at a concentration of $4.5 \mathrm{mg} \cdot \mathrm{L}^{-1}$ of sodium selenate under nonstress condition had an increase of $63.09 \%$ compared to the control. The lowest germination rate ( 0.33 seed per day) was observed under $1.2 \mathrm{MPa}$ stress and nonpriming (Fig. 2).

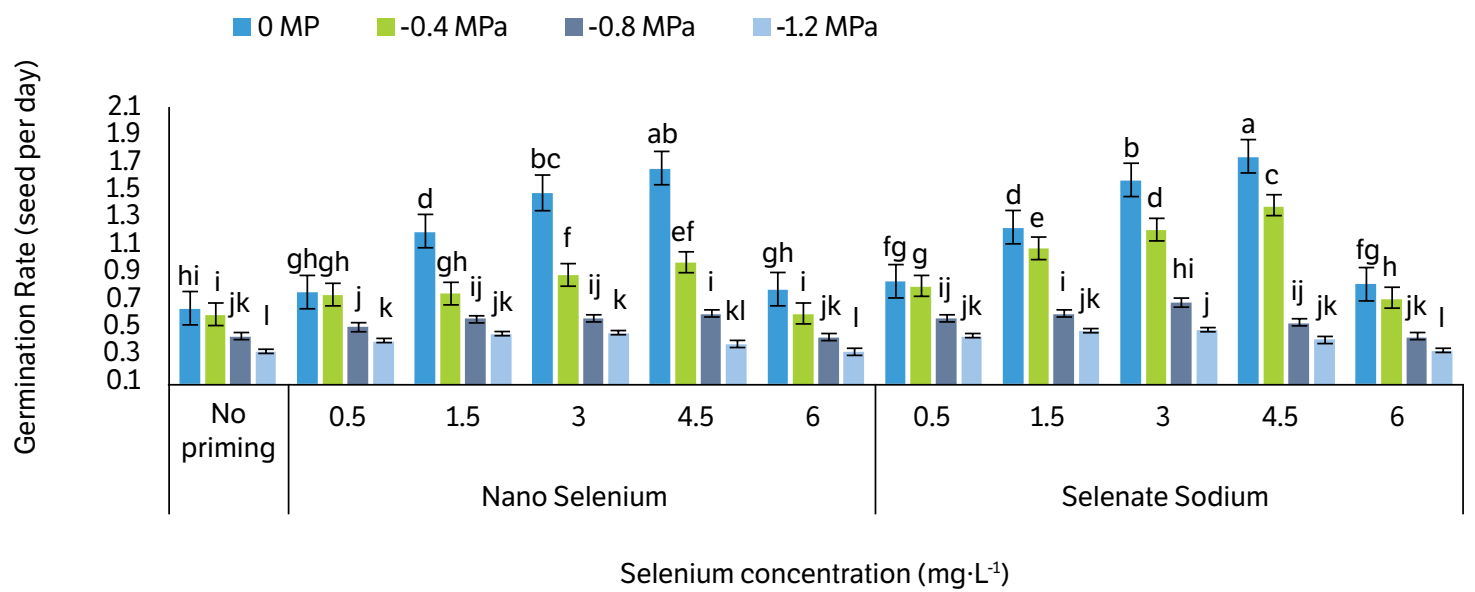

Figure 2. Comparison of different sodium selenate and selenium nanoparticles concentrations effects on germination rate of quinoa under drought stress conditions.

Note. Bars labelled with the same letters are not significantly different according to Duncan's multiple comparison test at $\mathrm{p}<0.05$. 


\section{Seedling length}

In this study, seedling length was affected by drought stress and by nutripriming with selenium. Drought stress significantly reduced seedling length. Selenium nanoparticles and sodium selenate at concentrations between 0.5 to $4.5 \mathrm{mg} \cdot \mathrm{L}^{-1}$ increased seedling length while higher concentration had a negative effect on seedling length. The greatest seedling length $(8.77 \mathrm{~cm})$ at a concentration of $4.5 \mathrm{mg} \cdot \mathrm{L}^{-1}$ of selenium nanoparticles under nonstress condition had an increase of $34.3 \%$ compared to the control. The lowest seedling length $(2.85 \mathrm{~cm}$ ) was observed under $-1.2 \mathrm{MPa}$ stress and nonpriming (Fig. 3 ).

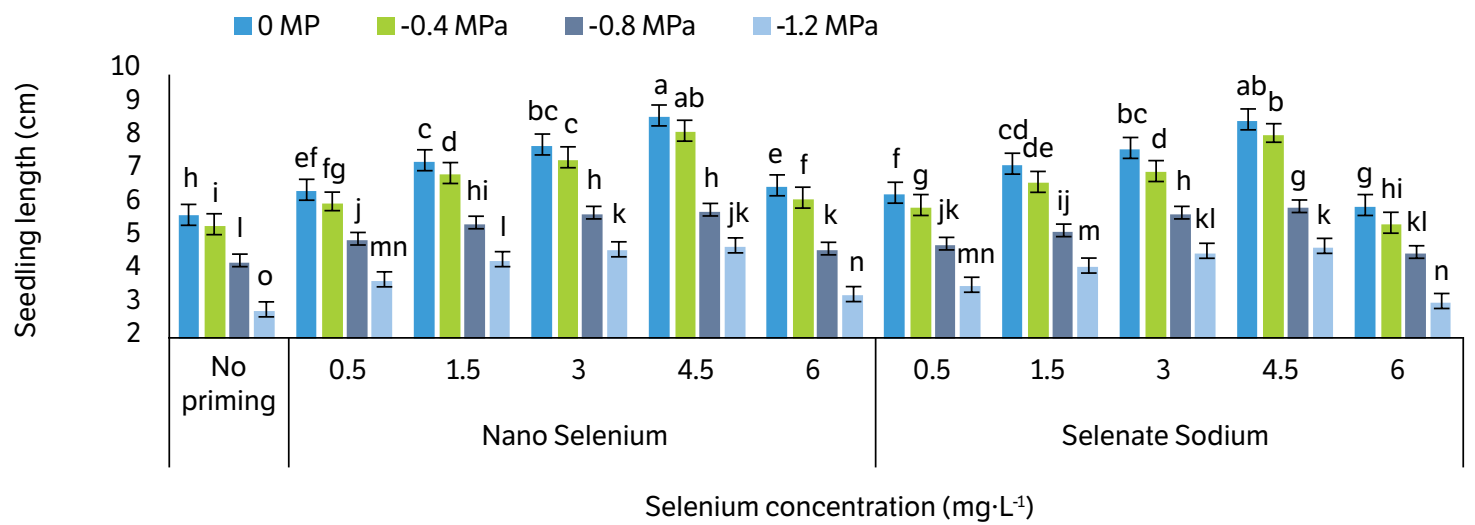

Figure 3. Comparison of different sodium selenate and selenium nanoparticles concentrations effects on seedling length of quinoa under drought stress conditions. Bars labelled with the same letters are not significantly different according to Duncan's multiple comparison test at $p<0.05$.

\section{Photosynthetic pigments content}

In this study, nutripriming with selenium and drought stress caused by PEG and the interaction of these treatments on chlorophyll a, chlorophyll b, total chlorophyll, and carotenoid content of quinoa seedlings were significant $(\mathrm{p} \leq 0.01)$ (Table 1$)$. Drought stress significantly reduced the content of photosynthetic pigments in quinoa seedlings. However, the content of photosynthetic pigments in quinoa seedlings primed with selenium was higher than on nonpriming (control) quinoa seeds. Concentrations above $3 \mathrm{mg} \cdot \mathrm{L}^{-1}$ of selenium nanoparticles accumulated chlorophyll and carotenoids. However, sodium selenate at concentrations above $3 \mathrm{mg} \cdot \mathrm{L}^{-1}$ significantly reduced the chlorophyll and carotenoid content. The highest photosynthetic pigment contents under nondrought stress conditions and nutripriming with selenium nanoparticles at concentrations of $6 \mathrm{mg} \cdot \mathrm{L}^{-1}\left(0.965 \mathrm{mg} \cdot \mathrm{g}^{-1} \mathrm{FW}\right)$ for chlorophyll a, $4.5 \mathrm{mg} \cdot \mathrm{L}^{-1}\left(1.002 \mathrm{mg} \cdot \mathrm{g}^{-1} \mathrm{FW}\right)$ for chlorophyll b, and $6 \mathrm{mg} \cdot \mathrm{L}^{-1}\left(1.94 \mathrm{mg} \cdot \mathrm{g}^{-1} \mathrm{FW}\right)$ for total chlorophyll and concentrations of $4.5 \mathrm{mg} \cdot \mathrm{L}^{-1}\left(2.33 \mathrm{mg} \cdot \mathrm{g}^{-1} \mathrm{FW}\right)$ for carotenoids, had an increase of $25.0,47.9,36.5$ and $43.4 \%$, respectively, compared to the control treatment.

Table 1. ANOVA results (mean square) of different selenium levels on some studied traits of quinoa under drought stress, Tehran, Iran.

\begin{tabular}{|c|c|c|c|c|c|c|c|c|}
\hline sov & df & $\begin{array}{l}\text { Germination } \\
\text { percentage }\end{array}$ & $\begin{array}{l}\text { Germination } \\
\text { rate }\end{array}$ & $\begin{array}{l}\text { Seedling } \\
\text { length }\end{array}$ & $\begin{array}{l}\text { Chlorophyll } \\
\text { a content }\end{array}$ & $\begin{array}{l}\text { Chlorophyll } \\
\text { b content }\end{array}$ & $\begin{array}{c}\text { Total } \\
\text { chlorophyll } \\
\text { content }\end{array}$ & $\begin{array}{l}\text { Carotenoid } \\
\text { content }\end{array}$ \\
\hline Drought stress (D) & 3 & $1752.79^{\star \star}$ & $1.454^{\star \star}$ & $51.841^{\star \star}$ & $0.7156^{\star \star}$ & $0.4553^{\star \star}$ & $2.3266^{\star \star}$ & $2.1716^{\star \star}$ \\
\hline Selenium (Se) & 1 & $110.50^{\star \star}$ & $1.3861^{\star \star}$ & $0.3616^{\star \star}$ & $0.14231^{\star \star}$ & $0.2548^{\star \star}$ & $0.7880^{\star \star}$ & $0.2793^{\star \star}$ \\
\hline $\begin{array}{c}\text { Selenium } \\
\text { concentration (C) }\end{array}$ & 5 & $911.100^{\star \star}$ & $14.217^{\star \star}$ & $14.217^{\star \star}$ & $0.14215^{\star \star}$ & $0.1810^{\star *}$ & $0.60000^{\star \star}$ & $0.4448^{\star \star}$ \\
\hline $\mathrm{D} \times \mathrm{Se}$ & 3 & $0.7936^{\star \star}$ & $1.447^{\star \star}$ & $1.447^{\star \star}$ & $0.0209^{\star *}$ & $0.0175^{\star \star}$ & $0.07734^{\star \star}$ & $0.0586^{\star \star}$ \\
\hline$D \times C$ & 15 & $15.052^{\star \star}$ & $0.6417^{\star \star}$ & $0.6417^{\star \star}$ & $0.0167^{\star \star}$ & $0.0157^{\star \star}$ & $0.04971^{\star \star}$ & 0.0179 ** \\
\hline $\mathrm{Se} \times \mathrm{C}$ & 5 & $17.322^{\star \star}$ & $0.1904^{*}$ & $0.1904^{\star \star}$ & $0.1073^{\star \star}$ & $0.1752^{\star \star}$ & $0.5524^{\star \star}$ & $0.2889^{\star *}$ \\
\hline $\mathrm{D} \times \mathrm{Se} \times \mathrm{C}$ & 15 & $4.800^{\star \star}$ & $0.3351^{\star \star}$ & $0.3351^{\star \star}$ & $0.0076^{\star \star}$ & $0.0159^{\star \star}$ & $0.0407^{\star \star}$ & $0.0261^{\star \star}$ \\
\hline Error & & 2.7301 & 0.08597 & 0.0859 & 0.0010 & 0.0008 & 0.0017 & 0.0036 \\
\hline CV (\%) & & 2.075 & 0.145 & 4.366 & 4.366 & 4.012 & 3.988 & 5.278 \\
\hline
\end{tabular}

${ }^{*}$ and ${ }^{* \star}$ indicate significant differences at $\mathrm{p} \leq 0.05$ and 0.01 , respectively. 


\section{Protein content}

In this experiment, drought stress treatment by PEG and priming with selenium and the interaction of these treatments showed significant effects $(\mathrm{p} \leq 0.01)$ on quinoa seedlings' protein content (Table 2). Nutripriming at selenium nanoparticle concentrations of 0.5 to $4.5 \mathrm{mg} \cdot \mathrm{L}^{-1}$ under nondrought stress conditions compared to control conditions (nonpriming) and severe stress $(-1.2 \mathrm{MPa})$, increased the protein content. However, priming with sodium selenate at concentrations higher than $3 \mathrm{mg} \cdot \mathrm{L}^{-1}$ significantly reduced the protein content under PEG-induced drought stress conditions. The results also showed the lowest protein content in the nonpriming and -1.2 MPa drought stress treatments (Table 3).

Table 2. ANOVA results (mean square) of different selenium levels on some studied traits of quinoa under drought stress, Tehran, Iran.

\begin{tabular}{ccccccc}
\hline SOV & df & Proline content & $\begin{array}{c}\text { Protein } \\
\text { content }\end{array}$ & CAT activity & APX activity & SOD activity \\
\hline Drought stress (D) & 3 & $19.7700^{\star \star}$ & $32.014^{\star \star}$ & $17.783^{\star \star}$ & $22.206^{\star \star}$ & $24.461^{\star \star}$ \\
\hline Selenium (Se) & 1 & $0.9958^{\star \star}$ & $0.4209^{\star}$ & $3.5022^{\star \star}$ & $1.2481^{\star \star}$ & $12.270^{\star \star}$ \\
\hline Selenium concentration (C) & 5 & $1.1640^{\star \star}$ & $2.5178^{\star \star}$ & $3.5091^{\star \star}$ & $4.2243^{\star \star}$ & $10.183^{\star \star}$ \\
\hline D $\times$ Se & 3 & $0.0245^{\star}$ & $0.0747^{\star}$ & $0.1353^{\star \star}$ & $0.1239^{\text {ns }}$ & $0.0487^{\text {ns }}$ \\
\hline D $\times$ C & 15 & $0.0693^{\star}$ & $0.0948^{\star}$ & $0.3185^{\star \star}$ & $0.2495^{\star \star}$ & $0.1254^{\star}$ \\
\hline Se $\times$ C & 5 & $1.1094^{\star \star}$ & $2.0748^{\star \star}$ & $1.2853^{\star \star}$ & $0.8268^{\star \star}$ & $2.5091^{\star \star}$ \\
\hline D $\times$ Se $\times$ C & 15 & $0.0585^{\star \star}$ & $0.0729^{\star \star}$ & $0.2155^{\star \star}$ & $0.0422^{\star \star}$ & $0.2090^{\star \star}$ \\
\hline Error & & 0.1776 & 0.0831 & 0.0631 & 0.0690 & 0.0612 \\
\hline CV $(\%)$ & & 4.799 & 5.446 & 4.693 & 5.961 & 2.586 \\
\hline
\end{tabular}

${ }^{n s}$ represents nonsignificant difference at the 0.05 level of probability. ${ }^{*}$ and ${ }^{* *}$ indicate significant differences at $p \leq 0.05$ and 0.01 , respectively.

\section{Proline content}

The data showed significant differences $(\mathrm{p} \leq 0.01)$ in the proline content of quinoa seedlings under drought caused by PEG and nutripriming with selenium (Table 2). Pretreatment with concentration higher than $3 \mathrm{mg} \cdot \mathrm{L}^{-1}$ of sodium selenate showed a decreasing effect on proline content. Therefore, the results indicate a low toxicity of selenium nanoparticles at high concentrations compared to the ionic form of sodium selenate (Table 3 ).

\section{Catalase (CAT) activity}

The results showed a significant effect $(\mathrm{p} \leq 0.01)$ of nutripriming with selenium and drought stress caused by PEG and the interaction effects of these treatments on CAT activity in quinoa seedlings (Table 2). In this experiment, however, with increasing levels of PEG-induced drought stress, CAT activity increased compared to no stress (control). This study showed a significant difference between different nutripriming levels with sodium selenate and selenium nanoparticles on CAT activity compared to nonpriming conditions. The maximum activity CAT at $4.5 \mathrm{mg}$ of selenium nanoparticles and $3 \mathrm{mg}$ of sodium selenate under $-1.2 \mathrm{MPa}$ stress condition increased by 83.9 and $76.4 \%$, respectively, compared to the control (Table 4).

\section{Superoxide dismutase (SOD) activity}

In this study, drought stress caused by PEG and nutripriming with different selenium levels and the interaction effects of these treatments on the activity of SOD in quinoa seedlings were significant (Table 2). With increasing drought stress levels, SOD activity showed an increasing trend compared to nondrought stress conditions. The data shows that the selenium toxicity threshold depends on the type and concentrations used. Thus, in this study the enzyme activity significantly increased with priming selenium nanoparticles at a concentration of 0.5 to $6 \mathrm{mg} \cdot \mathrm{L}^{-1}$ and with sodium selenate at 0.5 to $3 \mathrm{mg} \cdot \mathrm{L}^{-1}$. The highest SOD enzyme activity (4.03 unit $\cdot \mathrm{min}^{-1} \cdot \mathrm{mg}$ protein) at a concentration of $6 \mathrm{mg} \cdot \mathrm{L}^{-1}$ of selenium nanoparticles 
under -1.2 MPa stress condition had an increase of $68.4 \%$ compared to the control. The lowest SOD enzyme activity (1.27 unit $\cdot \mathrm{min}^{-1} \cdot \mathrm{mg}$ protein) was observed under the nonstress conditions and nonpriming (Table 4).

Table 3. Comparison of the mean effect of seed priming with several rates of selenium nanoparticles and sodium selenate on some physiological traits of quinoa under drought stress.

\begin{tabular}{|c|c|c|c|c|c|c|c|}
\hline \multicolumn{8}{|c|}{ Drought stress with PEG } \\
\hline & \multicolumn{4}{|c|}{ Chlorophyll a content $\left(\mathrm{mg} \cdot \mathrm{g}^{-1} \mathrm{FW}\right)$} & \multicolumn{3}{|c|}{ Chlorophyll b content $\left(\mathrm{mg} \cdot \mathrm{g}^{-1} \mathrm{FW}\right)$} \\
\hline & $0 \mathrm{MPa}$ & $-0.4 \mathrm{MPa}$ & $-0.8 \mathrm{MPa}$ & $-1.2 \mathrm{MPa}$ & $0 \mathrm{MPa}$ & $-0.4 \mathrm{MPa}$ & $-0.8 \mathrm{MPa}$ \\
\hline Control (nonpriming) & $0.723 \mathrm{~h}$ & $0.676 \mathrm{jk}$ & $0.589 n$ & $0.389 \mathrm{u}$ & $0.522 \mathrm{~g}$ & $0.402 \mathrm{j}$ & $0.274 \mathrm{mn}$ \\
\hline \multicolumn{8}{|l|}{ Nano selenium $\left(\mathrm{mg} \cdot \mathrm{L}^{-1}\right)$} \\
\hline 0.5 & $0.755 \mathrm{fg}$ & $0.727 \mathrm{~h}$ & $0.660 \mathrm{k}$ & $0.423 \mathrm{~s}$ & $0.532 \mathrm{~g}$ & $0.474 \mathrm{i}$ & 0.3121 \\
\hline 1.5 & $0.834 \mathrm{~d}$ & $0.793 \mathrm{e}$ & $0.673 \mathrm{jk}$ & $0.503 q$ & $0.733 \mathrm{~d}$ & $0.688 \mathrm{e}$ & $0.379 \mathrm{k}$ \\
\hline 3.0 & $0.876 \mathrm{bc}$ & $0.804 \mathrm{e}$ & $0.683 \mathrm{j}$ & $0.515 q$ & $0.884 \mathrm{~b}$ & 0.723 de & $0.381 \mathrm{k}$ \\
\hline 4.5 & $0.902 \mathrm{~b}$ & $0.849 \mathrm{~cd}$ & $0.685 j$ & 0.547 op & $1.003 \mathrm{a}$ & $0.735 \mathrm{~d}$ & $0.285 \mathrm{mn}$ \\
\hline 6.0 & $0.965 \mathrm{a}$ & $0.868 \mathrm{c}$ & 0.5510 & $0.388 \mathrm{u}$ & $0.732 \mathrm{~d}$ & $0.345 \mathrm{kl}$ & $0.264 n$ \\
\hline \multicolumn{8}{|l|}{ Sodium selenate $\left(\mathrm{mg} \cdot \mathrm{L}^{-1}\right)$} \\
\hline 0.5 & $0.744 \mathrm{~g}$ & 0.719 hi & $0.614 \mathrm{~m}$ & $0.406 \mathrm{t}$ & $0.476 \mathrm{i}$ & $0.412 \mathrm{j}$ & $0.340 \mathrm{kl}$ \\
\hline 1.5 & $0.784 \mathrm{f}$ & $0.737 \mathrm{gh}$ & 0.6391 & $0.457 \mathrm{rs}$ & 0.645 ef & 0.550 gef & $0.358 \mathrm{kl}$ \\
\hline 3.0 & $0.864 \mathrm{c}$ & $0.783 \mathrm{f}$ & $0.654 \mathrm{kl}$ & $0.488 r$ & $0.800 \mathrm{c}$ & 0.604 ef & $0.291 \mathrm{~m}$ \\
\hline 4.5 & $0.740 \mathrm{~g}$ & $0.703 i$ & $0.588 n$ & $0.369 \mathrm{vu}$ & $0.588 \mathrm{f}$ & $0.411 \mathrm{j}$ & $0.271 \mathrm{mn}$ \\
\hline \multirow[t]{2}{*}{6.0} & $0.734 \mathrm{gh}$ & $0.683 j$ & 0.541 op & $0.359 v$ & $0.432 \mathrm{ij}$ & 0.3121 & 0.245 no \\
\hline & \multicolumn{4}{|c|}{ Total chlorophyll content $\left(\mathrm{mg} \cdot \mathrm{g}^{-1} \mathrm{FW}\right)$} & \multicolumn{3}{|c|}{ Carotenoid content $\left.\left(\mathrm{mg} \cdot \mathrm{g}^{-1} \mathrm{FW}\right)\right)$} \\
\hline Control (nonpriming) & $1.23 \mathrm{f}$ & $1.05 \mathrm{~h}$ & $0.95 \mathrm{i}$ & $0.715 \mathrm{Im}$ & $1.32 \mathrm{de}$ & $1.23 \mathrm{e}$ & $1.08 \mathrm{f}$ \\
\hline \multicolumn{8}{|l|}{ Nano selenium $\left(\mathrm{mg} \cdot \mathrm{L}^{-1}\right)$} \\
\hline 0.5 & $1.26 \mathrm{f}$ & $1.08 \mathrm{~h}$ & $1.05 \mathrm{~h}$ & $0.772 \mathrm{kl}$ & $1.35 \mathrm{de}$ & $1.26 \mathrm{e}$ & 1.19 ef \\
\hline 1.5 & $1.36 \mathrm{de}$ & $1.22 \mathrm{f}$ & $1.08 \mathrm{~h}$ & $0.944 i$ & $1.66 \mathrm{c}$ & $1.53 \mathrm{~cd}$ & $1.21 \mathrm{e}$ \\
\hline 3.0 & $1.66 \mathrm{~cd}$ & $1.46 \mathrm{~d}$ & $1.28 \mathrm{f}$ & $0.810 \mathrm{k}$ & $1.88 \mathrm{~b}$ & $1.58 \mathrm{~cd}$ & $1.23 \mathrm{e}$ \\
\hline 4.5 & $1.94 \mathrm{~b}$ & $1.78 \mathrm{c}$ & $1.48 \mathrm{~d}$ & $1.019 \mathrm{~h}$ & $2.33 a$ & $1.75 \mathrm{bc}$ & $1.37 \mathrm{de}$ \\
\hline 6.0 & $2.36 a$ & $2.19 a b$ & $1.30 \mathrm{e}$ & $0.692 \mathrm{~m}$ & $1.66 \mathrm{c}$ & $1.38 \mathrm{de}$ & $1.05 \mathrm{f}$ \\
\hline \multicolumn{8}{|l|}{ Sodium selenate $\left(\mathrm{mg} \cdot \mathrm{L}^{-1}\right)$} \\
\hline 0.5 & $1.15 \mathrm{~g}$ & $1.04 \mathrm{~h}$ & $1.03 \mathrm{~h}$ & $0.735 I$ & $1.33 \mathrm{de}$ & $1.25 \mathrm{e}$ & $1.05 \mathrm{f}$ \\
\hline 1.5 & $1.30 \mathrm{e}$ & $1.20 \mathrm{f}$ & $1.05 \mathrm{~h}$ & $0.872 \mathrm{ijk}$ & $1.53 \mathrm{~cd}$ & $1.39 \mathrm{~d}$ & $1.22 \mathrm{e}$ \\
\hline 3.0 & $1.55 \mathrm{~d}$ & $1.38 \mathrm{e}$ & $1.14 \mathrm{~g}$ & $0.923 \mathrm{ij}$ & $1.76 \mathrm{bc}$ & $1.44 \mathrm{~d}$ & $1.32 \mathrm{de}$ \\
\hline 4.5 & $1.30 \mathrm{e}$ & $1.20 \mathrm{f}$ & $0.92 \mathrm{ij}$ & $0.710 \mathrm{Im}$ & $1.56 \mathrm{~cd}$ & $1.38 \mathrm{de}$ & $1.05 \mathrm{f}$ \\
\hline \multirow[t]{2}{*}{6.0} & $1.15 \mathrm{~g}$ & $1.01 \mathrm{~h}$ & $0.90 \mathrm{j}$ & $0.673 n$ & $1.38 \mathrm{de}$ & 1.13 ef & $0.91 \mathrm{~g}$ \\
\hline & \multicolumn{4}{|c|}{ Protein content (\%) } & \multicolumn{3}{|c|}{ Proline content $\left(\mu \mathrm{mol} \cdot \mathrm{g}^{-1} \mathrm{FW}\right)$} \\
\hline Control (nonpriming) & 2.21ef & $1.90 \mathrm{~g}$ & $1.25 \mathrm{jk}$ & $0.88 \mathrm{Im}$ & $0.94 \mathrm{rq}$ & 1.04 op & $1.92 \mathrm{i}$ \\
\hline \multicolumn{8}{|l|}{ Nano selenium } \\
\hline 0.5 & $2.35 \mathrm{e}$ & $2.32 \mathrm{e}$ & $1.65 \mathrm{hi}$ & $1.00 \mathrm{kl}$ & 1.08 op & 1.541 & $2.01 \mathrm{~h}$ \\
\hline 1.5 & $2.54 \mathrm{~cd}$ & $2.32 \mathrm{e}$ & $1.76 \mathrm{~h}$ & $1.10 \mathrm{k}$ & 1.230 & $1.69 \mathrm{k}$ & $2.09 \mathrm{~h}$ \\
\hline 3.0 & $2.84 \mathrm{~b}$ & $2.64 \mathrm{c}$ & $2.00 \mathrm{f}$ & $1.35 \mathrm{j}$ & $1.32 n$ & $1.71 \mathrm{jk}$ & $2.23 \mathrm{~g}$ \\
\hline 4.5 & $3.23 \mathrm{a}$ & $2.83 \mathrm{~b}$ & 2.29 ef & $1.37 \mathrm{j}$ & $1.36 \mathrm{n}$ & $1.73 \mathrm{jk}$ & $2.36 \mathrm{f}$ \\
\hline 6.0 & $2.63 c$ & $2.37 \mathrm{e}$ & $1.78 \mathrm{~h}$ & $1.00 \mathrm{kl}$ & $1.47 \mathrm{~m}$ & $1.82 \mathrm{j}$ & $2.85 \mathrm{c}$ \\
\hline \multicolumn{8}{|l|}{ Sodium selenate } \\
\hline 0.5 & 2.26 ef & 2.10 ef & $1.34 \mathrm{j}$ & $0.61 n$ & $1.00 p$ & $1.39 \mathrm{~m}$ & $1.95 \mathrm{j}$ \\
\hline 1.5 & $2.44 \mathrm{~d}$ & 2.26 ef & $1.66 \mathrm{hi}$ & 0.981 & 1.11 op & $1.51 \mathrm{I}$ & $2.09 \mathrm{~h}$ \\
\hline 3.0 & $2.74 \mathrm{~b}$ & $2.36 \mathrm{e}$ & $1.57 \mathrm{i}$ & 0.941 & $1.20 \circ$ & $1.56 I$ & $2.13 \mathrm{gh}$ \\
\hline 4.5 & $2.24 \mathrm{f}$ & $2.02 \mathrm{f}$ & $1.14 \mathrm{k}$ & $0.72 \mathrm{Im}$ & $0.98 \mathrm{q}$ & $1.13 \mathrm{op}$ & $1.94 \mathrm{i}$ \\
\hline 6.0 & $1.80 \mathrm{~g}$ & $1.57 i$ & $1.10 \mathrm{k}$ & $0.62 n$ & $0.93 \mathrm{rq}$ & 1.04 op & $1.67 \mathrm{k}$ \\
\hline
\end{tabular}

Note. Different letters mean significant differences between treatments according to the LSD test at $p \leq 0.05$. 
Table 4. Comparison of the mean effect of seed priming with several rates of selenium nanoparticles and sodium selenate on some physiological traits of quinoa under drought stress.

\begin{tabular}{|c|c|c|c|c|c|c|c|c|c|c|}
\hline \multicolumn{11}{|c|}{ Drought stress with PEG } \\
\hline \multirow{2}{*}{$\begin{array}{c}\text { Selenium } \\
\text { forms and } \\
\text { concentrations }\end{array}$} & \multicolumn{3}{|c|}{$\begin{array}{c}\text { CAT activity } \\
\left.\text { (U. } \text { min }^{-1} \cdot \mathrm{mg} \text { protein }\right)\end{array}$} & \multicolumn{3}{|c|}{$\begin{array}{c}\text { SOD activity } \\
\left.\text { (U. } \text { min }^{-1} \cdot \mathrm{mg} \text { protein }\right)\end{array}$} & \multicolumn{4}{|c|}{$\begin{array}{c}\text { APX activity } \\
\left.\text { (U. } \text { min }^{-1} \cdot \mathrm{mg} \text { protein }\right)\end{array}$} \\
\hline & $0 \mathrm{MPa}$ & $\begin{array}{l}-0.4 \\
\mathrm{MPa}\end{array}$ & $\begin{array}{l}-0.8 \\
\mathrm{MPa}\end{array}$ & $-1.2 \mathrm{MPa}$ & $0 \mathrm{MPa}$ & $\begin{array}{l}-0.4 \\
\mathrm{MPa}\end{array}$ & $\begin{array}{l}-0.8 \\
\mathrm{MPa}\end{array}$ & $-1.2 \mathrm{MPa}$ & $0 \mathrm{MPa}$ & $\begin{array}{l}-0.4 \\
\mathrm{MPa}\end{array}$ \\
\hline $\begin{array}{c}\text { Control } \\
\text { (non-priming) }\end{array}$ & 0.755 no & $1.19 \mid$ & $1.90 \mathrm{gh}$ & $2.07 \mathrm{~g}$ & $1.27 \mathrm{j}$ & $1.55 \mathrm{hi}$ & $2.08 \mathrm{f}$ & $2.65 \mathrm{~cd}$ & $0.266 I$ & $0.476 \mathrm{j}$ \\
\hline \multicolumn{11}{|l|}{ Nano selenium } \\
\hline 0.5 & $0.6543 \circ$ & $1.49 \mathrm{j}$ & $2.35 \mathrm{fg}$ & $2.45 \mathrm{f}$ & $1.32 \mathrm{ij}$ & $1.61 \mathrm{~h}$ & $2.06 \mathrm{f}$ & $2.53 \mathrm{~cd}$ & $0.985 \mathrm{gh}$ & $1.112 \mathrm{fg}$ \\
\hline 1.5 & $0.766 n$ & $1.54 \mathrm{j}$ & $2.65 \mathrm{e}$ & $2.87 \mathrm{~d}$ & $1.55 \mathrm{hi}$ & $1.95 \mathrm{fg}$ & $2.59 \mathrm{~cd}$ & $3.30 \mathrm{~b}$ & $1.002 \mathrm{~g}$ & $1.265 \mathrm{fg}$ \\
\hline 3.0 & $0.865 \mathrm{mn}$ & $1.72 \mathrm{hj}$ & $2.85 \mathrm{~d}$ & $3.05 \mathrm{~cd}$ & $1.79 \mathrm{gh}$ & $2.33 \mathrm{ef}$ & $2.76 \mathrm{~d}$ & $3.35 b$ & $1.043 \mathrm{~g}$ & 1.352 ef \\
\hline 4.5 & $0.9856 \mathrm{~m}$ & $1.80 \mathrm{~h}$ & $3.35 b$ & $4.70 \mathrm{a}$ & $1.88 \mathrm{~g}$ & $2.62 \mathrm{~cd}$ & $3.23 b c$ & $3.81 \mathrm{ab}$ & $1.332 \mathrm{ef}$ & $1.500 \mathrm{de}$ \\
\hline 6.0 & $1.0067 \mid$ & $1.30 \mathrm{k}$ & $1.85 \mathrm{gh}$ & $2.00 \mathrm{~g}$ & 2.13 ef & $2.96 \mathrm{c}$ & $3.24 b c$ & $4.03 a$ & $1.00 \mathrm{~g}$ & $1.232 \mathrm{fg}$ \\
\hline \multicolumn{11}{|l|}{ Sodium selenate } \\
\hline 0.5 & $0.8564 \mathrm{mn}$ & $1.42 \mathrm{k}$ & $2.24 \mathrm{fg}$ & $2.30 \mathrm{fg}$ & $1.28 \mathrm{j}$ & $1.55 \mathrm{hi}$ & $2.09 \mathrm{f}$ & $2.50 \mathrm{~cd}$ & $0.8778 \mathrm{~h}$ & $1.071 \mathrm{~g}$ \\
\hline 1.5 & 0.7554 no & $1.50 \mathrm{j}$ & $2.50 \mathrm{ef}$ & $2.85 \mathrm{~d}$ & $1.57 \mathrm{hi}$ & $1.84 \mathrm{~g}$ & $2.48 \mathrm{e}$ & $2.99 \mathrm{c}$ & $1.004 \mathrm{~g}$ & $1.221 \mathrm{fg}$ \\
\hline 3.0 & $0.8342 \mathrm{mn}$ & $1.70 \mathrm{i}$ & $2.65 \mathrm{e}$ & $3.20 \mathrm{c}$ & $1.68 \mathrm{~h}$ & $1.97 \mathrm{fg}$ & $2.49 \mathrm{e}$ & $3.30 \mathrm{~b}$ & $0.654 i$ & $0.952 \mathrm{gh}$ \\
\hline 4.5 & $0.9678 \mathrm{~m}$ & $1.38 \mathrm{k}$ & $1.97 \mathrm{~g}$ & $2.00 \mathrm{~g}$ & $1.56 \mathrm{hi}$ & $1.88 \mathrm{~g}$ & 2.37 ef & $2.81 \mathrm{~cd}$ & $0.4431 \mathrm{j}$ & $0.664 \mathrm{i}$ \\
\hline 6.0 & 0.7332 no & $0.93 \mathrm{~m}$ & $1.55 \mathrm{j}$ & $1.54 j$ & $1.11 \mathrm{k}$ & $1.47 \mathrm{i}$ & $1.69 \mathrm{~h}$ & $2.10 \mathrm{ef}$ & $0.2554 \mathrm{I}$ & $0.402 \mathrm{k}$ \\
\hline
\end{tabular}

Note. Different letters (APX, SOD, and APX) mean significant differences between treatments according to the LSD test at $p \leq 0.05$.

\section{Ascorbate peroxidase (APX) activity}

In this experiment, a significant effect of nutripriming with selenium and drought stress and on their interaction on APX activity in quinoa seedlings were observed (Table 2). Simultaneously, with the increase of drought stress levels, the APX activity showed an increasing trend. Nutripriming with selenium nanoparticles at a concentration of $4.5 \mathrm{mg} \cdot \mathrm{L}^{-1}$ and sodium selenate at a concentration of $1.5 \mathrm{mg} \cdot \mathrm{L}^{-1}$ under $-1.2 \mathrm{MPa}$ drought stress increased the enzymatic activity of APX compared to the control treatment (nonpriming) (Table 4). In general, priming with sodium selenate at high concentrations has a toxic effect and inhibits enzyme activity.

\section{DISCUSSION}

Because seed germination is the initial step of a plant's life cycle and the critical phase of seedling establishment, it is very sensitive to drought stress (Ibrahim 2016). The results of the present study showed that with increasing drought stress caused by PEG, germination percentage and germination rate have a decreasing trend (Fig 1 and 2). Drought stress disrupts metabolism due to low amylase activity, resulting in significant inhibition of seedling germination and growth (Farooq et al. 2020). Increasing the PEG concentration significantly reduced cottonseed germination and germination rate caused by negative regulation in physiological mechanisms and inhibited seed water absorption (Bai et al. 2020). Similar results were also reported in rice seedlings (Sohag et al. 2020). Drought stress has been shown in numerous studies to drastically reduce seed germination in a variety of plant species (Liu, J. et al., 2019; Liu, M. et al., 2018; Liu, X. et al., 2016).

The above findings support the idea that selenium seed priming improves quinoa seedling drought tolerance by increasing germination (Fig. 1) which confirms the findings of Nawaz et al. (2013), that selenium seed priming improves drought tolerance in wheat seedlings by increasing germination. Soaking lemon seeds (Citrus aurantifolia L.) in a solution of selenium nanoparticles at a concentration of $50 \mathrm{ppm}$ increased the germination percentage compared to the control (Ahmed 
et al. 2018). Seed priming increased de novo production of germination-promoting substances, membrane restructuring, the activity of hydrolytic enzymes, and decrease leakage of metabolites (Khaliq et al. 2015; Zeid et al. 2019). In the present study high selenium concentrations resulted in a significant percentage germination reduction and germination rate (Figs. 1 and 2). According to recent research, high selenium concentrations resulted in a significant germination reduction in cowpea (Lapaz et al. 2019). The inhibitory effect of selenium at high concentrations during germination may be due to the inhibition of the activity of enzymes that hydrolyze compounds necessary for embryonic growth (Khaliq et al. 2015; Sreekala and Lalitha 1998).

In this study, priming seeds with selenium, especially at low concentrations, was able to reduce the effects of drought stress to some extent and increased quinoa seedling growth. Similar results have been reported by soaking the seeds of cowpea with sodium selenate and nanoparticles. This increase in cowpea seedling growth could be attributed to the synthesis of some germination stimuli, increased plant cell division, an increase in some hydrolytic enzymes (amylase and protease) that stimulate antioxidant activity, and increased abilities for absorbing and use water (Zeid et al. 2019). High selenium concentrations resulted in a significant germination reduction on the growth of cowpea seedlings and decreased seedling length in rice (Das et al. 2019; Lapaz et al. 2019). In Vicia faba, the root length significantly impacted by application of selenium nanoparticle at concentrations of 10 and $20 \mathrm{ppm}$ (Zedan and Omar 2019).

With increasing drought stress due to PEG, the concentration of photosynthetic pigments was decreased compared to nonstress conditions. Similar results were also reported in wheat seedlings (Sattar et al. 2019). Production of ROS under drought stress leads to damage of chloroplasts and a significant reduction in chlorophyll content of leaves (Ahmadizadeh 2013). In the current study, chlorophyll contents increased with increased in selenium nanoparticle concentrations in the test seedlings, which confirms the findings of Moulick et al. (2017) in work with rice. With increasing concentration of sodium selenate, photosynthetic pigment levels decreased due to toxicity at concentrations above $3 \mathrm{mg} \cdot \mathrm{L}-1$ (Table 3). Similarly, high concentrations of sodium selenate reduced the content of photosynthetic pigments in cowpea (Lapaz et al. 2019) and rice seedlings (Das et al. 2019). Such a negative effect of selenium on chlorophyll content is due to its effect on porphobilinogen synthesis, which was also observed by Fargašová et al. (2006) in Sinapis alba L.

In the present study, PEG-induced drought stress led to higher proline accumulation. PEG-induced drought stress increased proline content in cotton seeds (Bai et al. 2020), rice (Sohag et al. 2020) and pearl millet seedlings (Awan et al. 2021) as observed in the current study. Proline is a key osmotic regulator and free radical scavenger that can reduce water potential and hence reduce stress damage (Hayat et al. 2012; Rathinasabapathi, 2000). In the present study, proline content increased with increasing concentrations of selenium nanoparticles and sodium selenate $(<3 \mathrm{mg} \cdot \mathrm{L}-1)$. Similarly, proline content in grape (Liu et al. 2019), and rice seedlings (Das et al. 2019) was observed with increasing the concentration of sodium selenate. Increased proline levels and activity of antioxidant enzymes by additional administration of selenium under water deficient conditions indicate that there is a relationship between the antioxidant system and proline accumulation (Nawaz et al. 2015b).

The findings of the present study showed that PEG-induced drought stress reduced soluble protein content. The decrease in soluble protein concentration in seedlings under water stress conditions could be attributed to increased protease enzyme activity (Ashraf 2004; Nawaz et al. 2015a). Priming quinoa seeds with selenium and sodium selenate nanoparticles at appropriate concentrations increased protein content. These results are similar to those of Zeid et al. (2019) in priming cowpea seeds with sodium selenate and selenium nanoparticles and those of Khaliq et al. (2015) in priming rice with sodium selenate and those of Oprică et al. (2018) in priming basil seedlings with selenium nanoparticles. Selenium priming increased the amount of protein in wheat under drought stress (Nawaz et al. 2013) and the amount of proline and protein in oilseeds (camellia and rapeseed) under water deficiency (Ahmad et al. 2021), which is consistent with the results of this study. Protein synthesis is reduced at high (toxic) concentrations of selenium. This may be due to selenium being replaced by sulfur in amino acids, resulting in disruption of selenoproteins and thus inactivation of proteins and enzymes (Terry et al. 2000; Van Hoewyk 2013).

Plants have a complex enzymatic defense system to combat oxidative damage and excessive ROS production (Ahammed et al. 2020; Souza et al. 2014). For example, SOD and POD are antioxidant defense systems that protect plasma membranes from peroxidation caused by an excess of oxygen free radicals (Xiao et al. 2019). The present study showed that severe 
PEG-induced drought plays a physiological role as a preoxidative agent and increases $\mathrm{H}_{2} \mathrm{O}_{2}$ concentration. Seed priming with selenium improved the activity of antioxidant enzymes such as CAT, SOD and ascorbate at this level of stress to reduce $\mathrm{H}_{2} \mathrm{O}_{2}$ to $\mathrm{H}_{2} \mathrm{O}$, which is consistent with the findings of Ahmad et al. (2021) with oilseed crops. Drought stress inhibited SOD activity in cotton seeds (Bai et al. 2020), Which does not support the present research findings. Similar to this study, pretreatment of cucumber seedlings with selenium increased APX activity under water stress (Jóźwiak and Politycka 2019). Treatment with selenium nanoparticles increased enzymes activities such as APX, SOD in tomatoes, which is also in accord with the current study (Hernández-Hernández et al. 2019). In Oprică et al. (2018), treatment with selenium nanoparticles reduced CAT activity in basil seedlings which was not in line with the findings of the current study. According to the doses of selenium used in this experiment, application of selenium increases the activity of SOD and APX compared to the control, as observed in cowpea by Silva et al. (2018). Pretreatment of grape seedlings with selenium increased the activity of CAT and SOD enzymes compared to the control (Liu et al. 2019).

\section{CONCLUSION}

These results indicate that seed priming with appropriate concentrations of selenium nanoparticles or sodium selenate under drought stress, can improve germination percentage as well as enhance physiological reactions such as photosynthetic pigments, proline, protein and antioxidant enzymes (CAT, SOD, APX) levels in quinoa seedlings. The present study also provides physiological evidence that priming with selenium nanoparticles has a more positive effect than sodium selenate. Thus, selenium nanoparticles and sodium selenate could represent a good alternative to improve quinoa crop productivity under drought stress, especially for the production of higher quality crops. However, further studies should be conducted under field conditions to determine the appropriate selenium concentration in quinoa seeds under drought stress. In addition, further field research in different environments and soil types is needed to determine the potential of seleniumimpregnated treatments to improve drought tolerance of quinoa.

\section{AUTHORS' CONTRIBUTION}

Conceptualization: Gholami S. and Amini Dehaghi M.; Methodology: Gholami S. and Rezazadeh A.; Investigation: Gholami S.; Writing - Original Draft: Gholami S.; Writing - Review and Editing: Gholami S., Amini Dehaghi M. and Naji A. M.; Funding Acquisition: Amini Dehaghi M.; Resources, Amini Dehaghi M. and Naji A. M.; Supervision: Amini Dehaghi M.

\section{DATA AVAILABILITY STATEMENT}

All data sets were generated or analyzed in the current study.

\section{FUNDING}

Shahed University of Tehran

\section{ACKNOWLEDGMENTS}

Not applicable. 


\section{REFERENCES}

Ahammed, G. J., Wang, Y., Mao, Q., Wu, M., Yan, Y., Ren, J., Wang, X., Liu, A. and Chen, S. (2020). Dopamine alleviates bisphenol A-induced phytotoxicity by enhancing antioxidant and detoxification potential in cucumber. Environmental Pollution, 259, 113957. https://doi. org/10.1016/j.envpol.2020.113957

Ahmad, Z., Anjum, S., Skalicky, M., Waraich, E. A, Tariq, R. M. S., Ayub, M. A., Hossain, A., Hassan, M. M., Brestic, M., Islam, M. S., HabibUr-Rahman, M., Wasaya, A., lqbal, M. A. and El Sabagh, A. (2021). Selenium alleviates the adverse effect of drought in oilseed crops camelina (Camelina sativa L.) and canola (Brassica napus L.). Molecules, 26, 1699. https://doi.org/10.3390/molecules26061699

Ahmadizadeh, M. (2013). Physiological and agro-morphological response to drought stress. Middle-East Journal of Scientific Research, 13, 998-1009.

Ahmed, H. S., Ahmed, M. F., Shoala, T. and Salah, M. (2018). Impact of single or fractionated radiation and selenium nano-particles on acid lime (Citrus aurantifolia L.) seed germination ability and seedlings growth. Advances in Agriculture and Environmental Science: Open Access, 1, 91-100. https://doi.org/10.30881/aaeoa.00016

[AOSA] Association of Official Seed Analysts. (1990). Rules for testing seeds. Association of Official Seed Analysts, Ithaca, New York.

Arnon, D. I. (1949). Copper enzymes in isolated chloroplasts: Polyphenoloxidase in Beta vulgaris. Plant Physiology, 24, 1-15. https://doi. org/10.1104/pp.24.1.1

Ashraf, M. (2004). Some important physiological selection criteria for salt tolerance in plants. Flora-Morphology, Distribution, Functional Ecology of Plants, 199, 361-376. https://doi.org/10.1078/0367-2530-00165

Awan, S. A., Khan, I., Rizwan, M., Zhang, X., Brestic, M., Khan, A., El-Sheikh, M. A., Alyemeni, M. N., Ali, S. and Huang, L. (2021). Exogenous abscisic acid and jasmonic acid restrain polyethylene glycol induced drought by improving the growth and antioxidative enzyme activities in pearl millet. Physiologia Plantarum, 172, 809-819. https://doi.org/10.1111/ppl.13247

Bai, Y., Xiao, S., Zhang, Z., Zhang, Y., Sun, H., Zhang, K., Wang, X., Bai, Z., Li, C. and Liu, L. (2020). Melatonin improves the germination rate of cotton seeds under drought stress by opening pores in the seed coat. Plant Biology, 8, e9450. https://doi.org/10.7717/peerj.9450

Bates, L. S., Waldren, R. P. and Teare, I. D. (1973). Rapid determination of free proline for water-stress studies. Plant and Soil, 39, 205207. https://doi.org/10.1007/BF00018060

Bradford, M. M. (1976). A rapid and sensitive method for the quantitation of microgram quantities of protein utilizing the principle of protein-dye binding. Analytical Biochemistry, 72, 248-254. https://doi.org/10.1016/0003-2697(76)90527-3

Cai, K., Chen, X., Han, Z., Wu, X., Zhang, S., Li, Q., Nazir, M. M., Zhang, G. and Zeng, F. (2020). Screening of worldwide barley collection for drought tolerance: The assessment of various physiological measures as the selection criteria. Frontiers in Plant Science, $11,1159$. https://doi.org/10.3389/fpls.2020.01159

Chen, R. J., Wang, L. X., Zhang, X. J., Wang, S. L., Li, H. and Gao, S. J. (2020). Physiological and molecular basis for the effects of exogenous selenium application on wheat seedling performance under drought stress. Applied Ecology and Environmental Research, 18, 12271236. https://doi.org/10.15666/aeer/1801_12271236

Das, D., Seal, P. and Biswas, A. K. (2019). Influence of selenium on growth, antioxidants production and physiological parameters of rice (Oryza sativa L.) seedlings and its possible reversal by coapplication of sulphate. American Journal of Plant Sciences, 10, $2236-2278$. https://doi.org/10.4236/ajps.2019.1012158

Dawood, M. G. (2018). Stimulating plant tolerance against abiotic stress through seed priming. In A. Rakshi and H. B. Singh (Eds.), Advances in Seed Priming (p. 147-183). Singapore: Springer. https://doi.org/10.1007/978-981-13-0032-5_10 
Delatorre-Herrera, J., Rojas-Urrutia, J., Rojo, L. E. and Graf, B. L. (2019). Osmotic stress in Chenopodium quinoa Willd.: Variations in osmoprotectants at different phenological stages. African Journal of Agricultural Research, 14, 361-368.

Djanaguiraman, M., Belliraj, N., Bossmann, S. H. and Prasad, P. V. V. (2018). High-temperature stress alleviation by selenium nanoparticle treatment in grain sorghum. ACS Omega, 3, 2479-2491. https://doi.org/10.1021/acsomega.7b01934

Du, Z. and Bramlage, W. J. (1994). Superoxide dismutase activities in senescing apple fruit (Malus domestica Borkh.). Journal of Food Science, 59, 581-584. https://doi.org/10.1111/j.1365-2621.1994.tb05567.x

Fargašová, A., Pastierová, J. and Svetkova, K. (2006). Effect of Se-metal pair combinations (Cd, Zn, Cu, Pb) on photosynthetic pigments production and metal accumulation in Sinapis alba L. seedlings. Plant, Soil and Environment, 52, 8-15. https://doi.org/10.17221/3340-PSE

Farooq, M., Romdhane, L., Al Sulti, M. K. R. A., Rehman, A., Al Busaidi, W. M. and Lee, D.-J. (2020). Morphological, physiological and biochemical aspects of osmopriming induced drought tolerance in lentil. Journal of Agronomy and Crop Science, 206, 176-186. https:// doi.org/10.1111/jac.12384

Farooq, M., Ullah, A., Rehman, A., Nawaz, A., Nadeem, A., Wakeel, A., Nadeem, F. and Siddique, K. H. M. (2018). Application of zinc improves the productivity and biofortification of fine grain aromatic rice grown in dry seeded and puddled transplanted production systems. Field Crops Research, 216, 53-62. https://doi.org/10.1016/j.fcr.2017.11.004

Graf, B. L., Rojo, L. E., Delatorre Herrera, J., Poulev, A., Calfio, C. and Raskin, I. (2016). Phytoecdysteroids and flavonoid glycosides among Chilean and commercial sources of Chenopodium quinoa: Variation and correlation to physico chemical characteristics. Journal of the Science of Food and Agriculture, 96, 633-643. https://doi.org/10.1002/jsfa.7134

Gordillo-Bastidas, E., Díaz-Rizzolo, D. A., Roura, E., Massanés, T. and Gomis, R. (2016). Quinoa (Chenopodium quinoa Willd.), from nutritional value to potential health benefits: An integrative review. Journal of Nutrition and Food Sciences, 6, 497. https://doi. org/10.4172/2155-9600.1000497

Hajihashemi, S., Skalicky, M., Brestic, M. and Pavla, V. (2020). Cross-talk between nitric oxide, hydrogen peroxide and calcium in salt-stressed Chenopodium quinoa Willd. At seed germination stage. Plant Physiology and Biochemistry, 154, 657-664. https://doi. org/10.1016/j.plaphy.2020.07.022

Hassan, M. J., Raza, M. A., Khan, I., Meraj, T. A., Ahmed, M., Shah, G. A., Ansar, M., Awan, S. A., Khan, N., Iqbal, N., Peng, Y. and Li, Z. (2020). Selenium and salt interactions in black gram (Vigna mungo $L$ ): Ion uptake, antioxidant defense system, and photochemistry efficiency. Plants, 9, 467. https://doi.org/10.3390/plants9040467

Hayat, S., Hayat, Q., Alyemeni, M. N., Wani, A. S., Pichtel, J. and Ahmad, A. (2012). Role of proline under changing environments: A review. Plant Signaling \& Behavior, 7, 1456-1466. https://doi.org/10.4161/psb.21949

Hernández-Hernández, H., Quiterio-Gutiérrez, T., Cadenas-Pliego, G., Ortega-Ortiz, H., Hernández-Fuentes, A. D., Cabrera de la Fuente, M., Valdés-Reyna, J. and Juárez-Maldonado, A. (2019). Impact of selenium and copper nanoparticles on yield, antioxidant system, and fruit quality of tomato plants. Plants, 8, 355. https://doi.org/10.3390/plants8100355

Hossain, A., Skalicky, M., Brestic, M., Maitra, S., Sarkar, S., Ahmad, Z., Vemuri, H., Garai, S., Mondal, M., Bhatt, R., Kumar, P., Banerjee, P., Saha, S., Islam, T. and Laing, A. M. (2021). Selenium biofortification: roles, mechanisms, responses and prospects. Molecules, $26,881$. https://doi.org/10.3390/molecules26040881

Ibrahim, E. A. (2016). Seed priming to alleviate salinity stress in germinating seeds. Journal of Plant Physiology, 192, 38-46. https://doi. org/10.1016/j.jplph.2015.12.011

[ISTA] International Seed Testing Association. (2008). International Rules for Seed Testing. International. Bassersdorf: ISTA.

Jiang, C., Zu, C., Lu, D., Zheng, Q., Shen, J., Wang, H. and Li, D. (2017). Effect of exogenous selenium supply on photosynthesis, Na+ accumulation and antioxidative capacity of maize (Zea mays L.) under salinity stress. Scientific Reports, 7, 42039. https://doi.org/10.1038/srep42039 
Jóźwiak, W. and Politycka, B. (2019). Effect of selenium on alleviating oxidative stress caused by a water deficit in cucumber roots. Plants, 8, 217. https://doi.org/10.3390/plants8070217

Khaliq, A., Aslam, F., Matloob, A., Hussain, S., Geng, M., Wahid, A. and ur Rehman, H. (2015). Seed priming with selenium: Consequences for emergence, seedling growth, and biochemical attributes of rice. Biological Trace Element Research, 166, 236-244. https://doi. org/10.1007/s12011-015-0260-4

Khan, M. I. R., Nazir, F., Asgher, M., Per, T. S. and Khan, N. A. (2015). Selenium and sulfur influence ethylene formation and alleviate cadmium-induced oxidative stress by improving proline and glutathione production in wheat. Journal of Plant Physiology, 173, 9-18. https://doi.org/10.1016/j.jplph.2014.09.011

Khan, I., Raza, M. A., Awan, S. A., Shah, G. A., Rizwan, M., Ali, B., Tariq, R., Hassan, M. J., Alyemeni, M. N., Brestic, M., Zhang, X., Ali, S. and Huang, L. (2020). Amelioration of salt induced toxicity in pearl millet by seed priming with silver nanoparticles (AgNPs): The oxidative damage, antioxidant enzymes and ions uptake are major determinants of salt tolerant capacity. Plant Physiology and Biochemistry, 156, 221-232. https://doi.org/10.1016/j.plaphy.2020.09.018

Lapaz, A. M., Santos, L. F. M., Yoshida, C. H. P., Heinrichs, R., Campos, M. and Reis, A. R. (2019). Physiological and toxic effects of selenium on seed germination of cowpea seedlings. Bragantia, 78, 498-508. https://doi.org/10.1590/1678-4499.20190114

Li, C.-x., Feng, S.-I., Shao, Y., Jiang, L.-n., Lu, X.-y. and Hou, X.-I. (2007). Effects of arsenic on seed germination and physiological activities of wheat seedlings. Journal of Environmental Sciences, 19, 725-732. https://doi.org/10.1016/S1001-0742(07)60121-1

Liopa-Tsakalidi, A., Kaspiris, G., Salahas, G. and Barouchas, P. (2012). Effect of salicylic acid (SA) and gibberellic acid (GA3) pre-soaking on seed germination of stevia (Stevia rebaudiana) under salt stress. Journal of Medicinal Plants Research, 6, 416-423. https://doi. org/10.5897/JMPR11.1106

Liu, J., Hasanuzzaman, M., Wen, H., Zhang, J., Peng, T., Sun, H. and Zhao, Q. (2019). High temperature and drought stress cause abscisic acid and reactive oxygen species accumulation and suppress seed germination growth in rice. Protoplasma, 256, 1217-1227. https:// doi.org/10.1007/s00709-019-01354-6

Liu, M., Wang, Z., Xiao, H.-m., and Yang, Y. (2018). Characterization of TaDREB1 in wheat genotypes with different seed germination under osmotic stress. Hereditas, 155, 26. https://doi.org/10.1186/s41065-018-0064-6

Liu, X., Hu, P., Huang, M., Tang, Y., Li, Y., Li, L. and Hou, X. (2016). The NF-YC-RGL2 module integrates GA and ABA signalling to regulate seed germination in Arabidopsis. Nature Communications, 7, 12768. https://doi.org/10.1038/ncomms12768

Magwanga, R. O., Lu, P., Kirungu, J. N., Dong, Q., Hu, Y., Zhou, Z., Cai, X., Wang, X., Hou, Y., Wang, K., Liu, F. (2018). Cotton late embryogenesis abundant (LEA2) genes promote root growth and confer drought stress tolerance in transgenic Arabidopsis thaliana. G3 Genes|Genomes|Genetics, 8, 2781-2803. https://doi.org/10.1534/g3.118.200423

Manjunatha, S. B., Biradar, D. P. and Aladakatti, Y. R. (2016). Nanotechnology and its applications in agriculture: A review. Journal Farm Science, 29, 1-13.

Moulick, D., Santra, S. C. and Ghosh, D. (2017). Seed priming with Se alleviate As induced phytotoxicity during germination and seedling growth by restricting As translocation in rice (Oryza sativa L cv IET-4094). Ecotoxicology and Environmental Safety, 145, 449-456. https:// doi.org/10.1016/j.ecoenv.2017.07.060

Nakano, Y. and Asada, K. (1981). Hydrogen peroxide is scavenged by ascorbate-specific peroxidase in spinach chloroplasts. Plant and Cell Physiology, 22, 867-880. https://doi.org/10.1093/oxfordjournals.pcp.a076232

Nawaz, F., Ahmad, R., Ashraf, M. Y., Waraich, E. A. and Khan, S. Z. (2015a). Effect of selenium foliar spray on physiological and biochemical processes and chemical constituents of wheat under drought stress. Ecotoxicology and Environmental Safety, 113, 191-200. https:// doi.org/10.1016/j.ecoenv.2014.12.003 
Nawaz, F., Ashraf, M. Y., Ahmad, R., Waraich, E. A., Shabbir, R. N. and Bukhari, M. A. (2015b). Supplemental selenium improves wheat grain yield and quality through alterations in biochemical processes under normal and water deficit conditions. Food Chemistry, 175, 350-357. https://doi.org/10.1016/j.foodchem.2014.11.147

Nawaz, F., Ashraf, M. Y., Ahmad, R. and Waraich, E. A. (2013). Selenium (Se) seed priming induced growth and biochemical changes in wheat under water deficit conditions. Biological Trace Element Research, 151, 284-293. https://doi.org/10.1007/s12011-012-9556-9

Nowak, V., Du, J. and Charrondière, U. R. (2016). Assessment of the nutritional composition of quinoa (Chenopodium quinoa Willd.). Food chemistry, 193, 47-54. https://doi.org/10.1016/j.foodchem.2015.02.111

Oprică, L., Molchan, O. and Grigore, M.-N. (2018). Salinity and selenium nanoparticles effect on antioxidant system and malondialdehyde content in Ocimum basilicum L. seedlings. Journal of Experimental and Molecular Biology, 19, 99-106.

Panuccio, M. R., Jacobsen, S. E., Akhtar, S. S., and Muscolo, A. (2014). Effect of saline water on seed germination and early seedling growth of the halophyte quinoa. AoB PLANTS, 6, plu047. https://doi.org/10.1093/aobpla/plu047

Pereira, E., Encina-Zelada, C., Barros, L., Gonzales-Barron, U., Cadavez, V., and Ferreira, I. C. F. R. (2019). Chemical and nutritional characterization of Chenopodium quinoa Willd. (quinoa) grains: A good alternative to nutritious food. Food Chemistry, 280, 110-114. https://doi.org/10.1016/j.foodchem.2018.12.068

Qureshi, M. K., Munir, S., Shahzad, A. N., Rasul, S., Nouman, W. and Aslam, K. (2018). Role of reactive oxygen species and contribution of new players in defense mechanism under drought stress in rice. International Journal of Agriculture and Biology, 20, 1339-1352. https:// doi.org/10.17957/IJAB/15.0640

Rathinasabapathi, B. (2000). Metabolic engineering for stress tolerance: Installing osmoprotectant synthesis pathways. Annals of Botany, 86, 709-716. https://doi.org/10.1006/anbo.2000.1254.

Reis, A. R., El-Ramady, H., Santos, E. F., Gratão, P. L. and Schomburg, L. (2017). Overview of selenium deficiency and toxicity worldwide: Affected areas, selenium-related health issues, and case studies. In E. A. H Pilon-Smits, L. H. E. Winkel and Z-Q. Lin (Eds.), Selenium in plants (p. 209-230). Cham: Springer. https://doi.org/10.1007/978-3-319-56249-0_13

Sajedi, N. A. and Madani, H. (2018). Comparison of physiological and biochemical responses of wheat and barley to Selenium by spraying application under rain fed conditions. Iranian Journal of Plant Physiology, 8, 2381-2389.

Sattar, A., Cheema, M. A., Sher, A., ljaz, M., Ul-Allah, S., Nawaz, A., Abbas, T. and Ali, Q. (2019). Physiological and biochemical attributes of bread wheat (Triticum aestivum L.) seedlings are influenced by foliar application of silicon and selenium under water deficit. Acta Physiologiae Plantarum, 41, 146. https://doi.org/10.1007/s11738-019-2938-2

Sheikhalipour, M., Esmaielpour, B., Behnamian, M., Gohari, G., Giglou, M. T., Vachova, P., Ratogi, A., Brestic, M. and Skalicky, M. (2021). Chitosan-selenium nanoparticle (Cs-Se NP) foliar spray alleviates salt stress in bitter melon. Nanomaterials, $11,684$. https://doi.org/10.3390/nano11030684

Shivay, Y. S., Prasad, R., Kaur, R. and Pal, M. (2016). Relative efficiency of zinc sulphate and chelated zinc on zinc biofortification of rice grains and zinc use-efficiency in Basmati rice. Proceedings of the National Academy of Sciences, India Section B: Biological Sciences, 86, 973-984. https://doi.org/10.1007/s40011-015-0544-7

Silva, V. M., Boleta, E. H. M., Lanza, M. G. D. B., Lavres, J., Martins, J. T., Santos, E. F., Santos, F. L. M., Putti, F. F., Furlani Junior, E., White, P. J., Broadley, M. R., Carvalho, H. W. P. and Reis, A. R. (2018). Physiological, biochemical, and ultrastructural characterization of selenium toxicity in cowpea plants. Environmental and Experimental Botany, 150, 172-182.

Sohag, A. A. M., Tahjib-Ul-Arif, M., Brestic, M., Afrin, S., Sakil, M. A., Hossain, M. T., Hossain, M. A. and Houssain, M. A. (2020). Exogenous salicylic acid and hydrogen peroxide attenuate drought stress in rice. Plant, Soil and Environment, 66, 7-13. https://doi. org/10.17221/472/2019-PSE 
Souza, T. C., Magalhães, P. C., Castro, E. M., Carneiro, N. P., Padilha, F. A. and Gomes Júnior, C. C. (2014). ABA application to maize hybrids contrasting for drought tolerance: Changes in water parameters and in antioxidant enzyme activity. Plant Growth Regulation, 73, 205217. https://doi.org/10.1007/s10725-013-9881-9

Sreekala, M., and Lalitha, K. (1998). Selenium-mediated differential response of -glucosidase and -galactosidase of germinating Trigonella foenum-graecum. Biological Trace Element Research, 64, 247-258. https://doi.org/10.1007/BF02783341

Terry, N., Zayed, A. M., Souza, M. P. and Tarun, A. S. (2000). Selenium in higher plants. Annual Review of Plant Physiology and Plant Molecular Biology, 51, 401-432. https://doi.org/10.1146/annurev.arplant.51.1.401

Thabet, S. G., Moursi, Y. S., Karam, M. A., Graner, A. and Alqudah, A. M. (2018). Genetic basis of drought tolerance during seed germination in barley. PloS ONE, 13, e0206682. https://doi.org/10.1371/journal.pone.0206682

Ul-Allah, S., lqbal, M., Maqsood, S., Naeem, M., ljaz, M., Ashfaq, W. and Hussain, M. (2018). Improving the performance of bread wheat genotypes by managing irrigation and nitrogen under semi-arid conditions. Archives of Agronomy and Soil Science, 64, 1678-1689. https://doi.org/10.1080/03650340.2018.1450974

Ullah, A., Shahzad, B., Tanveer, M., Nadeem, F., Sharma, A., Lee, D. J. and Rehman, A. (2019). Abiotic stress tolerance in plants through pre-sowing seed treatments with mineral elements and growth regulators. In M. Hasanuzzaman and V. Fotopoulos (Eds.), Priming and Pretreatment of Seeds and Seedlings (p. 427-445). Singapore: Springer.

Upadhyaya, H., Begum, L., Dey, B., Nath, P. K. and Panda, S. K. (2017). Impact of calcium phosphate nanoparticles on rice plant. Journal of Plant Science and Phytopathology, 1, 1-10. https://doi.org/10.29328/journal.jpsp.1001001

Van Hoewyk, D. (2013). A tale of two toxicities: Malformed selenoproteins and oxidative stress both contribute to selenium stress in plants. Annals of Botany, 112, 965-972. https://doi.org/10.1093/aob/mct163

Xia, X., Ling, L. and Zhang, W.-x. (2017). Genesis of pure Se(0) nano-and micro-structures in wastewater with nanoscale zero-valent iron (nZVI). Environmental Science: Nano, 4, 52-59. https://doi.org/10.1039/C6EN00231E

Xiao, S., Liu, L., Wang, H., Li, D., Bai, Z., Zhang, Y., Sun, H., Zhang, K. and Li, C. (2019). Exogenous melatonin accelerates seed germination in cotton (Gossypium hirsutum L.). PloS ONE, 14, e0216575. https://doi.org/10.1371/journal.pone.0216575

Zedan, A. and Omar, S. (2019). Nano selenium: Reduction of severe hazards of atrazine and promotion of changes in growth and gene expression patterns on Vicia faba seedlings. African Journal of Biotechnology, 18, 502-510. https://doi.org/10.5897/AJB2019.16773

Zeid, I. M., Gharib, F. A. E. L., Ghazi, S. M. and Ahmed, E. Z. (2019). Promotive effect of ascorbic acid, gallic acid, selenium and nanoselenium on seed germination, seedling growth and some hydrolytic enzymes activity of cowpea (Vigna unguiculata) seedling. Journal Plant Physiology \& Pathology, 7.

Zhang, J. and Kirkham, M. B. (1994). Drought-stress-induced changes in activities of superoxide dismutase, catalase, and peroxidase in wheat species. Plant and Cell Physiology, 35, 785-791. https://doi.org/10.1093/oxfordjournals.pcp.a078658

Zhang, M., Tang, S., Huang, X., Zhang, F., Pang, Y., Huang, Q., and Yi, Q. (2014). Selenium uptake, dynamic changes in selenium content and its influence on photosynthesis and chlorophyll fluorescence in rice (Oryza sativa L.). Environmental and Experimental Botany, 107, 39-45. https://doi.org/10.1016/j.envexpbot.2014.05.005 